\title{
Las nuevas TIC y los métodos educativos tradicionales (MET) en la educación para la salud bucal en estudiantes de Básica Primaria
}

\author{
Tomás Bernal-Álvarez ${ }^{\star}, \mathrm{MsC}_{{ }_{1}}$, Yhinny Paola Santos-Machado, MsC. (c) ${ }_{2}$, Teresita Alzate-Yepes, PhD. , \\ María Eugenia Morales-Mora, MsC. , Andrés A. Agudelo-Suárez, PhD. , Adriana Posada-López, MsC., \\ Alejandro Martínez-Londoño, Esp. (c)
}

Universidad de Antioquia, Facultad de Odontología, Medellín, Colombia ${ }_{2}$ Universidad Nacional de La Plata, La Plata, Argentina

${ }_{3}$ Universidad de Antioquia, Escuela de Nutrición y Dietética, Medellín, Colombia ${ }_{4}$ Universidad de las Islas Baleares, Mallorca, España

${ }_{5}$ Universidad Cooperativa de Colombia, Facultad de Odontología, Medellín, Colombia

Recibido: 11 de septiembre del 2014. Aprobado: 21 de octubre del 2014.

*Autor de correspondencia: Tomás Bernal Álvarez. Universidad de Antioquia, Facultad de Odontología. Calle 64 n. 5 52-59, Medellín, Colombia. Teléfono: (57) 4 2196772. Correo electrónico: tbernala@gmail.com

Cómo citar este artículo: Bernal-Álvarez T, Santos-Machado YP, Alzate-Yepes T, Morales-Mora ME, Agudelo-Suárez AA, Posada-López A, Martínez-Londoño A. Las nuevas TIC y los Métodos Educativos Tradicionales (MET) en la educación para la salud bucal en estudiantes de Básica Primaria. Rev Nac 0dontol. 2014;10(19):15-22. doi: http://dx.doi.org/10.16925/od.v10i19.842

Resumen. Introducción: se pretende evidenciar los efectos mediadores de las nuevas TIC y los métodos educativos tradicionales (MET), en el tema del cuidado bucal, por medio de indicadores de higiene bucal, conocimientos, actitudes y prácticas escolares en estudiantes de Básica Primaria de la Institución Educativa Francisco Miranda, Medellín. Métodos: estudio evaluativo a través de elementos de un estudio cuantitativo y descriptivo con datos de tres momentos en el tiempo: primer momento, marzo de 2011 (momento base o línea base); segundo momento, mayo de 2011 (luego de implementar MET); tercer momento, noviembre de 2011 (luego de implementar NTIC). Se hicieron exámenes clínicos para indicadores de higiene bucal (IHO) y encuestas a escolares sobre conocimientos, actitudes y prácticas respecto al cuidado bucal. Resultados: existe una mejoría en conocimientos y prácticas de escolares participantes del estudio, la cual se visibilizó en el segundo momento, al implementar los мET. Se presentó un cambio positivo en la higiene bucal, en la clasificación buena, de 10 a 46\%, e igualmente positiva en la clasificación regular y mala, de 66 a 44\% y de 24 a 10\%, respectivamente. El uso de NTIC puede arrojar resultados positivos en aprendizajes y conocimientos de hábitos de higiene bucal, siempre y cuando estas sean constantes y motivadoras. Conclusiones: los indicadores observados permitieron evaluar positivamente los resultados del estudio; se invita al fortalecimiento de estrategias de Educación para la Salud (EpS) mediadas por NTIC para aprendizajes cognoscitivos significativos y por MET para aprendizaje y desarrollo de habilidades prácticas significativas, en proyectos educativos institucionales (PEI).

Palabras clave: higiene bucal, educación para la salud, educación en salud dental, nuevas tecnologías de la información y la comunicación.

New ICT and Traditional Educational Methods (TEM) in Oral Health Education among Basic Primary Students

Abstract. Introduction: The aim is to show the mediating effects of new ICT and traditional educational methods (тем) in the field of oral health, using indicators for oral hygiene, knowledge, attitudes and school practices Basic Primary Students at the Francisco Miranda Educational Institution in Medellín. Methods: an evaluative study using quantitative and descriptive elements with data from three moments in time: first moment, March 2011 (base moment or baseline); second moment, May 2011 (after тем implementation); third moment November 2011 (after implementing NICT). Clinical examinations were performed for oral hygiene indicators (IHo from the Spanish acronym) and surveys were applied among the schoolchildren about oral health knowledge, attitudes and practices. Results: There has been an improvement in the knowledge and practices of primary school students participating in the study, which was evident during the second moment, which followed implementation of the тем. There was a positive change in oral health, with those classified as good going from $10 \%$ to $46 \%$, as well as among those classified as middling or deficient, increasing from $44 \%$ to $66 \%$ and from $10 \%$ to $24 \%$, respectively. The use of NICT can have positive results in learning and knowledge of oral health habits, as long as it is constant and motivating. Conclusions: The indicators observed led to a positive assessment of the results of the study; the study invites us to strengthen Health Education strategies using NICT for significant cognitive learning and those using TЕм for learning and developing significant practical abilities in institutional educational projects (PEI from the Spanish acronym).

Keywords: oral hygiene, health education, dental health education, new information and communication technologies. 


\section{As novas TIC e os métodos educativos tradicionais (MET) na educação para a saúde bucal em estudantes de Educação Ensino Fundamental}

Resumo. Introdução: pretende-se evidenciar os efeitos mediadores das novas TIC e os métodos educativos tradicionais (MET), no tema do cuidado bucal, por meio de indicadores de higiene bucal, conhecimentos, atitudes e práticas escolares em estudantes de Educação Ensino Fundamental da Instituição Educativa Francisco Miranda, Medellín. Métodos: estudo avaliativo através de elementos de um estudo quantitativo e descritivo com dados de três momentos no tempo: primeiro momento, março de 2011 (momento base ou linha base); segundo momento, maio de 2011 (depois de implementar MET); terceiro momento, novembro de 2011 (depois de implementar NTIC). Fizeram-se exames clínicos para indicadores de higiene bucal (IHO) e entrevistas aos escolares sobre conhecimentos, atitudes e práticas a respeito do cuidado bucal. Resultados: existe uma melhoria em conhecimentos e práticas de escolares participantes do estudo, a qual se visibilizó no segundo momento, implementado o MET. Apresentou-se uma mudança positiva na higiene bucal, na classificação boa, de 10 a $46 \%$, e igualmente positiva na classificação regular e má, de 66 a $44 \%$ e de 24 a 10\%, respectivamente. O uso de NTIC pode produzir resultados positivos em aprendizagens e conhecimentos de hábitos de higiene bucal, desde que estas sejam constantes e motivadoras. Conclusões: os indicadores observados permitiram avaliar positivamente os resultados do estudo; convida-se ao fortalecimento de estratégias de Educação para a Saúde (Eps) mediadas por NTIC para aprendizagens cognoscitivas significativas e por MET para aprendizagem e desenvolvimento de habilidades práticas significativas, em projetos educativos institucionais (PEI)

Palavras-chave: higiene bucal, educação para a saúde, educação em saúde dental, novas tecnologias da informação e comunicação.

\section{Introducción}

La Organización Mundial de la Salud (oms), en el contexto de la Carta de Ottawa, destaca la importancia de la eps como "arma de transformación del ser humano, la cual debería darse con el compromiso del sector educativo y de la salud al aplicar metodologías para informar y educar sobre actitudes y formas de vida más saludables" [1]

Por tal motivo, se requiere la consideración, la introducción, la adopción y el desarrollo de nuevas estrategias y metodologías educativas que impacten positivamente a la sociedad [2,3]. En la actualidad, las prácticas, las actitudes y los conocimientos en salud bucal juegan un papel importante en el desarrollo físico, psicológico y social del niño en sus primeros años de vida y en su etapa formativa.

Sin embargo, hay diferentes factores o determinantes [4] que afectan a dicha población, tales como educación, capacidad económica, condiciones de salud, ubicación geográfica, cultura, creencias y la misma sociedad. Estos factores pueden constituirse en potencializadores del buen desarrollo del ser humano en su calidad de vida $[1,5]$ o en factores de riesgo que impactan de manera negativa el progreso individual y social. De allí que se desarrollen estrategias diversas mediante procesos educativos adecuados en promoción de estilos de vida saludable, que se concretan en prácticas apropiadas, y en conocimientos y actitudes frente a la salud bucal, al ser este el objetivo de la Eps.
En este orden de ideas, la oms plantea que la salud es un factor fundamental en el desarrollo de los pueblos y la define como "un estado de completo bienestar físico, mental y social y no solo la ausencia de afecciones o enfermedades", y complementa expresando que la salud es un "componente fundamental del proceso de desarrollo humano, que se concibe como un proceso y un producto social dirigido a la creación de condiciones de bienestar para todos y por todos" [1].

Actualmente, los escolares están rodeados por un mundo que los invita a la tecnología para socializar, aprender y entretenerse. Entonces, es facultad del personal involucrado en la Eps aprovechar las diversas estrategias que ofrece el medio actual, a fin de ejercer como mediadores para promover estilos de vida saludable en la población escolar.

Para Vygotsky, la mediación es la tenencia de acceso directo a los objetos, el cual que es mediado a través de las herramientas de que dispone el estudiante para aprender. Es decir, que el conocimiento se elabora mediante la interacción con los demás, partiendo de los principios y valores establecidos de la cultura, y de las herramientas que dicha cultura le brinda al ser humano en cada época, desarrollada histórica y socialmente (por ejemplo, la educación en la virtualidad se vale de las herramientas tecnológicas para educar y formar a sus estudiantes) [6].

De acuerdo con la oms [1], en la educación tradicional se proponen diferentes métodos de educación para la salud, los cuales son clasificados según la relación entre el educando y el educado. De este modo, 
se define un grupo en el que se encuentra el método unidireccional directo, que está dirigido a individuos o grupos entre los que existe una relación directa de emisor-receptor, y se utiliza la palabra hablada con el apoyo de técnicas didácticas y medios o recursos técnicos [7].

En contraste, se entiende entonces que las NTIC son mediaciones que permiten la interacción con la información que ofrecen los ambientes virtuales, y por tal razón, constituyen un instrumento de enseñanza-aprendizaje que le posibilita al estudiante aprender a partir de la experiencia digital. En este sentido, la interacción juega un papel importante en la experiencia educativa, ya que permite el desarrollo del pensamiento, el conocimiento, las prácticas y las actitudes frente al objeto de conocimiento, con el fin de que tales experiencias sean significativas para la vida desde los procesos de interiorización y empoderamiento.

Por consiguiente, el objetivo de este estudio es evaluar los MET y las NTIC como mediadores en la educación para la salud bucal, articulados con estrategias de promoción de la salud y prevención de la enfermedad, teniendo en cuenta: 1) los conocimientos y las prácticas en salud bucal en escolares; y 2) la situación de higiene bucal de la población escolar de la Institución Educativa Francisco Miranda IEFM, con el fin de prevenir condiciones de deterioro que se manifiestan a causa de una inadecuada higiene bucal $[8,9]$.

\section{Métodos}

En el 2010, se inició el desarrollo de un proyecto más amplio, con el propósito de llevar a cabo una propuesta de investigación-acción por medio de la cual se contribuya a mejorar los niveles de conocimiento y práctica alrededor de lo odontológico, que redunden en beneficio de las condiciones de salud propias y de las familias de los estudiantes de la IEFM. En este marco, se pretendía revisar el efecto mediador de los MET y de las NTIC en los procesos de enseñanza y aprendizaje de actitudes, hábitos y prácticas relacionados con salud bucal.

En este orden de ideas, el grupo investigador ejecutó varias actividades. Para el uso de las NTIC (tercer momento), se hizo una presentación interactiva con diapositivas y una charla sobre la importancia de nuestra boca (con énfasis en las funciones estéticas, fonéticas, digestivas, masticatorias y su respectivo cuidado). Se presentó, a su vez, un video sobre el proceso de las enfermedades bucales inducidas por la placa bacteriana: caries, halitosis, gingivitis, periodontitis y cálculos dentales; después, los estudiantes pudieron demostrar los conocimientos adquiridos en un quiz multimedial. Se efectuó, además, una charla con participación interactiva, en la que se trabajaron conceptos y se respondieron preguntas.

Asimismo, se presentó un video sobre la manera correcta de eliminar la placa bacteriana, con énfasis en el cepillo de dientes, la seda dental, la crema dental y el flúor; y se realizó un afianzamiento del tema con la ayuda de una guía interactiva de cepillado [10], por medio de un modelo en Flash ${ }^{\circledR}$.

Luego, los estudiantes crearon un avatar en el portal City Salud [11]. Su objetivo era avanzar por diferentes escenarios de una ciudad del futuro, mediante la resolución de una serie de pruebas sobre hábitos saludables, para lo cual fue necesaria la lectura y comprensión de textos guía que ampliaban la información.

En la implementación de los MET (segundo momento), se desarrolló una actividad lúdico-educativa llamada "tren de la salud bucal", que constaba de cuatro "vagones" con diferentes ejes temáticos y objetivos como: identificar qué es la placa bacteriana y cómo se forma, demostrar las habilidades y los conocimientos en el uso del cepillo dental, reconocer el uso de la seda dental e identificar las ventajas del uso del flúor para la protección de los dientes.

De igual modo, con los MET, se hizo énfasis en el afianzamiento de las prácticas de higiene bucal por medio del control de placa; por su parte, el uso de las NTIC estaba dirigido a afianzar o reforzar los conocimientos de manera que los participantes pudieran llegar a ser agentes promotores de salud bucal desde ellos mismos y para enseñar a sus familias. Aquí, los estudiantes participaron en un foro en la plataforma Moodle, llamado "Enfermedades bucales en tu familia", en el que exponían un caso familiar de enfermedades inducidas por placa bacteriana, y aportaban qué hacer frente a los casos expuestos por los compañeros.

Luego, los estudiantes crearon un video educativo en Windows Movie Maker sobre una de las enfermedades bucales inducidas por placa bacteriana, además de un cartel para difusión en redes sociales sobre la prevención de una de estas enfermedades.

Esta es una investigación de carácter evaluativo que utiliza elementos de un estudio descriptivo desarrollado en tres momentos del tiempo: primer momento, en marzo de 2011 (momento base o línea base); segundo momento, en mayo de 2011 (luego de implementar MET), y tercer momento, en 
septiembre de 2011 (luego de implementar NTIC). La población de estudio estuvo conformada por escolares de 7 a 16 años de la IEFM, localizada en la comuna 4 de Medellín. Para el análisis, se utilizó la información de 22 escolares que participaron en los tres momentos de la investigación general. En la figura 1, se describe con detalle el proceso de participación de la población incluida en el estudio.

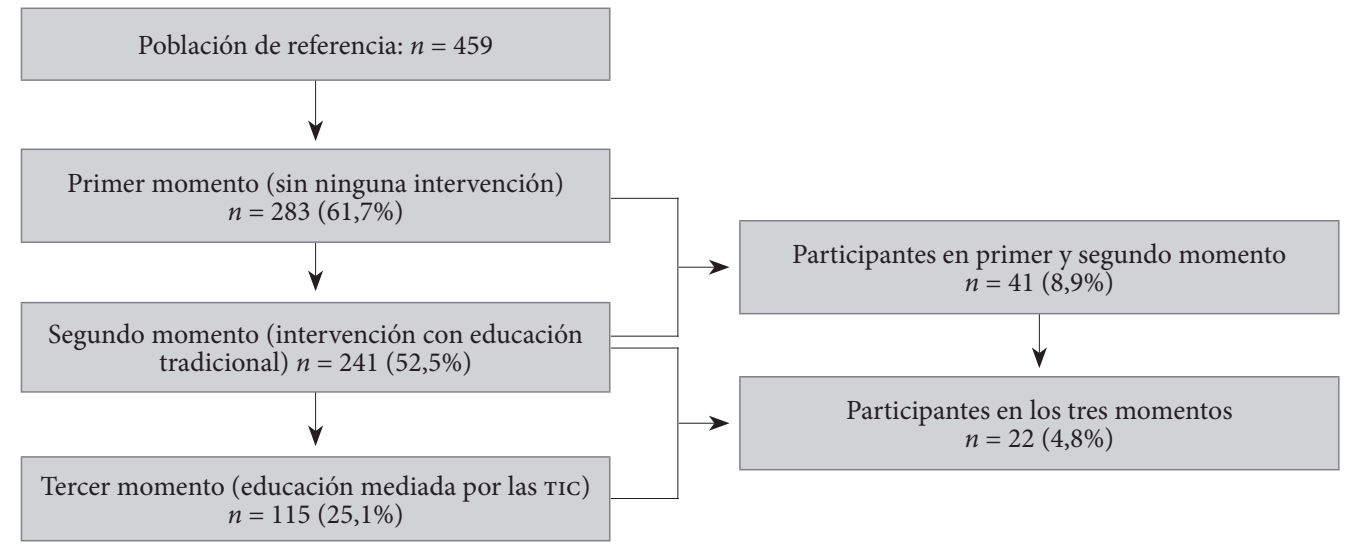

Figura 1. Diagrama de flujo sobre el proceso de recolección de información de la población de estudio. Medellín, 2012 Fuente: elaboración propia

En el trabajo de campo, se aplicaron varios instrumentos de recolección de información:

1) Examen clínico: índice de higiene oral simplificado de Green y Vermillon (IHOS), que es un indicador que mide la presencia de placa blanda en dientes deciduos y permanentes; el promedio que se registra va en un rango de 0 a 3,0 (a mayor promedio, más presencia de placa).

2) Encuesta sobre prácticas en salud bucal: el grupo investigador diseñó y aplicó un instrumento (disponible si se solicita a los autores) con información sociodemográfica básica, utilización de elementos para la higiene bucal y visita al odontólogo.

Concretamente, en este estudio se tuvieron en cuenta las siguientes variables: elementos de higiene bucal (cepillo de dientes, crema dental, seda dental, enjuague bucal), frecuencia de cepillado (veces/día), cepillado nocturno (siempre, algunas veces, pocas veces), visita al odontólogo/año (ninguna, una, dos).

Para el análisis descriptivo de las variables, en el caso del IHos se calculó el promedio con su intervalo de confianza al 95\% (IC 95\%) y la mediana con su correspondiente Rango Intercuartílico (RIC), para cada uno de los tres momentos según sexo. Después, se calculó en porcentaje el tipo de cambio en las condiciones de higiene bucal (positivo, negativo o no cambio).

Según la normativa internacional (Declaración de Helsinki) [4] y nacional (Resolución 008430 de 1993 del Ministerio de Salud) [12] sobre investigación en seres humanos, se considera que el presente estudio tiene riesgo mínimo, dado que se realiza un registro de datos a través de procedimientos comunes, consistentes en: características físicas, psicológicas o sociales de los pacientes seleccionados en el estudio. Se obtuvo consentimiento de las directivas de la institución, de los padres o adultos significativos, y de los escolares para participar en el estudio, y para protección de la identidad de la población entrevistada, la confidencialidad y la veracidad de la información.

\section{Resultados}

En la tabla 1, se presentan los datos sociodemográficos y las prácticas en higiene bucal en la población de estudio. El promedio de edad de la población investigada fue de $10,4( \pm 0,9)$ años, con el $64 \%$ mujeres. Todos los participantes usaban cepillo para su higiene bucal y enjuagues en menor proporción (45\%). La mitad de los escolares se cepillaban tres veces al día, y el $60 \%$ lo hacía antes de acostarse. El 30\% no ha visitado al odontólogo en el último año. 
Tabla 1. Descripción de las variables del estudio. Medellín, 2012 $(n=22)^{\mathrm{a}}$

\begin{tabular}{|c|c|c|}
\hline Variables & Número & $\%$ \\
\hline \multicolumn{3}{|l|}{ Sexo } \\
\hline Hombre & 8 & 36,4 \\
\hline Mujer & 14 & 63,6 \\
\hline \multicolumn{3}{|l|}{ Elementos higiene bucal $(\mathrm{Si})^{b}$} \\
\hline Cepillo de dientes & 20 & 100,0 \\
\hline Crema dental & 18 & 90,0 \\
\hline Seda dental & 11 & 55,0 \\
\hline Enjuague bucal & 9 & 45,0 \\
\hline \multicolumn{3}{|c|}{ Frecuencia de cepillado (veces/día) } \\
\hline Una & 1 & 5,0 \\
\hline Dos & 4 & 20,0 \\
\hline Tres & 10 & 50,0 \\
\hline Cuatro o más & 5 & 25,0 \\
\hline \multicolumn{3}{|l|}{ Cepillado antes de acostarse } \\
\hline Siempre & 12 & 60,0 \\
\hline Algunas veces & 5 & 25,0 \\
\hline Pocas veces & 3 & 15,0 \\
\hline \multicolumn{3}{|l|}{ Visita al odontólogo (año) } \\
\hline Ninguna & 6 & 30,0 \\
\hline Una & 7 & 35,0 \\
\hline Dos & 7 & 35,0 \\
\hline Total & 22 & 100,0 \\
\hline
\end{tabular}

a Datos perdidos: $(n=2)$, excepto para sexo.

b Porcentajes no excluyentes, basados en las respuestas a cada ítem.
$\mathrm{Al}$ analizar los promedios y las medianas en los tres momentos del estudio (figura 2 y tabla 2), se observa que hubo una disminución en las cifras del iHos entre el momento 2 y el momento 1, la cual fue mucho mayor en las mujeres; sin embargo, tanto el promedio como la mediana del IHos aumentaron en el momento 3.

Cuando se analizan cambios específicos (tabla 3), se observa que en el $63 \%$ de los hombres y en el $71 \%$ de las mujeres hubo un cambio positivo en las condiciones de higiene oral entre el momento 2 y el momento 1 . En segundo lugar, entre el momento 3 y el momento 1 el cambio positivo se observó en la cuarta parte de los hombres y en el $43 \%$ de las mujeres. Por último, entre el momento 3 y el momento 2 una cuarta parte de los hombres y el $29 \%$ de las mujeres tuvieron un cambio positivo en las condiciones de higiene oral.

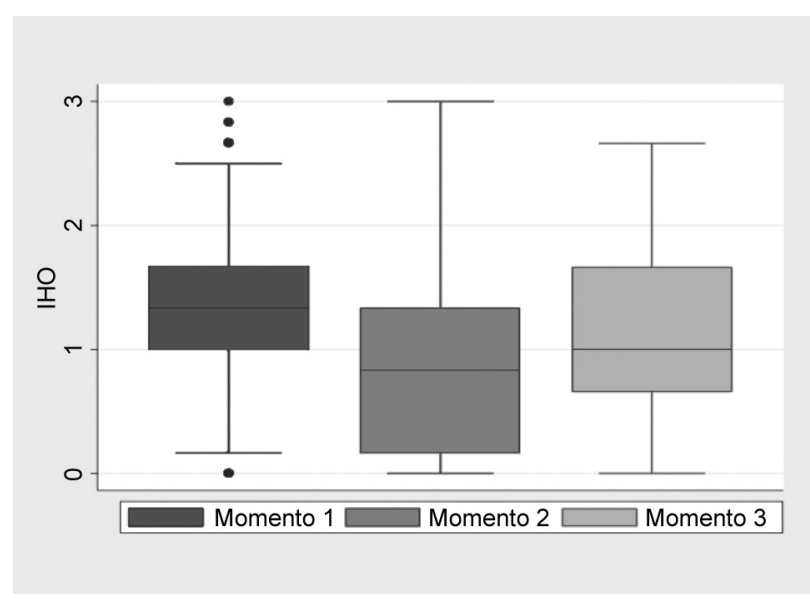

Figura 2. IHOs general de los escolares sin características. IEFM, Medellín, 2011

Fuente: elaboración propia

Tabla 2. Índice de higiene oral simplificado según sexo, Medellín, $2012(n=22)$

\begin{tabular}{|c|c|c|c|c|c|c|}
\hline \multirow{3}{*}{ Variables } & \multicolumn{6}{|c|}{ Iноs } \\
\hline & \multicolumn{3}{|c|}{ Promedio (IC 95\%) } & \multicolumn{3}{|c|}{ Mediana (RIC) } \\
\hline & Momento 1 & Momento 2 & Momento 3 & Momento 1 & Momento 2 & Momento 3 \\
\hline \multicolumn{7}{|l|}{ Sexo } \\
\hline Hombres & $0,88(0,49-1,26)$ & $0,48(0,25-1,29)$ & $1,28(0,63-1,94)$ & $0,67(0,67-1,13)$ & $0,00(0,00-1,04)$ & $1,23(0,79-1,71)$ \\
\hline Mujeres & $1,12(0,75-1,48)$ & $0,74(0,25-1,29)$ & $1,31(0,94-1,67)$ & $1,08(0,75-1,33)$ & $0,33(0,13-1,42)$ & $1,33(0,66-1,75)$ \\
\hline Total & $1,03(0,77-1,29)$ & $0,67(0,29-1,04)$ & $1,30(1,00-1,59)$ & $0,92(0,67-1,33)$ & $0,33(0,00-1,21)$ & $1,32(0,66-1,71)$ \\
\hline
\end{tabular}

Fuente: elaboración propia 
Tabla 3. Cambio en las condiciones de higiene bucal de acuerdo con IHos, Medellín, $2012(n=22)$

\begin{tabular}{|l|c|c|c|c|c|c|c|c|c|c|}
\hline \multirow{2}{*}{ Variables } & \multirow{2}{*}{ Muestra } & \multicolumn{9}{|c|}{ Cambio (porcentaje) } \\
\cline { 3 - 11 } & & \multicolumn{2}{|c|}{ Momento 2 - Momento 1 } & \multicolumn{2}{c|}{ Momento 3 - Momento 1 } & \multicolumn{3}{c|}{ Momento 3 - Momento 2 } \\
\cline { 3 - 11 } & Positivo & Negativo & $\begin{array}{c}\text { No } \\
\text { cambio }\end{array}$ & Positivo & Negativo & $\begin{array}{c}\text { No } \\
\text { cambio }\end{array}$ & Positivo & Negativo & $\begin{array}{c}\text { No } \\
\text { cambio }\end{array}$ \\
\hline Sexo & & & & & & & & & & \\
\hline Hombres & 8 & 62,5 & 25,0 & 12,5 & 25,0 & 75,0 & 0,0 & 25,0 & 75,0 & 0,0 \\
\hline Mujeres & 14 & 71,4 & 28,6 & 0,0 & 42,9 & 50,0 & 7,1 & 28,6 & 64,3 & 7,1 \\
\hline Total & 22 & $\mathbf{6 8 , 2}$ & 27,3 & 4,5 & 36,4 & 59,1 & 4,5 & 27,3 & $\mathbf{6 8 , 5}$ & 4,5 \\
\hline
\end{tabular}

Fuente: elaboración propia

\section{Discusión}

Los resultados principales de este estudio dan cuenta de que los participantes tienen prácticas de higiene bucal y visitan periódicamente al odontólogo. En cuanto a los índices de higiene oral, se observa un cambio positivo al implementar el uso de los MET, pero no así al implementar el uso de las NTIC.

En la literatura científica, se han evidenciado numerosas técnicas para hacer promoción de la salud. Su esencia radica en motivar a las personas para el autocuidado en salud bucal, a partir de una rutina diaria en la que primen materiales simples. Con dicha rutina, se generan cambios en el conocimiento y en las prácticas, y además surge el compromiso y la concepción de prevenir más que curar [13].

Es así que el objetivo de la educación se logra siempre y cuando se empleen los métodos apropiados, teniendo en cuenta las características de cada grupo, particularmente cuando se trabaja con población infantil. Todo lo anterior se sustenta en la definición de las funciones esenciales de la salud pública, cuando menciona como eje fundamental la información, la educación y el empoderamiento de las personas en temas de salud [14].

$\mathrm{Al}$ analizar los resultados encontrados en relación con los conocimientos y hábitos de los escolares de este estudio, se encontró que gran parte de la población escolar de la IEFM utiliza los implementos básicos de higiene oral. Sin embargo, el IHos se encuentra en nivel medio y disminuyó con la utilización de los MET.

Estos resultados coinciden con una intervención educativa realizada en un municipio de Venezuela, en el 2006, en 66 escolares entre 6 y 10 años; dicho estudio arrojó resultados positivos cuando se enseñó a los niños sobre las enfermedades bucales y las medidas preventivas, ya que el cambio en el nivel de conocimiento pasó de ser malo a bueno en un 34\% [15].

De igual manera, se reportaron resultados similares en un estudio en Cuba, en el 2013, en 40 alumnos de 7 a 11 años con retraso mental leve. A pesar de que es una población que se considera especial, se evidenció un incremento en el nivel de conocimientos y en el índice de higiene bucal. Además, el estado de salud bucal mejoró en términos cualitativos, pues no hubo evidencia de aparición de nuevas caries [16].

Posiblemente, el escenario de la escuela sea un campo favorable para que dichas intervenciones sean positivas. El estudio de Zanchett en Brasil [17] consideró que "la escuela es el campo propicio para la educación en salud". El eje central de la intervención desarrollada en los niños escolares fue la motivación, a fin de mejorar los hábitos de higiene oral y los nutricionales, por lo cual se reportó un impacto positivo en el IHOs.

Según lo anterior, las técnicas tradicionales han sido exitosas, en contraste con el presente estudio que muestra resultados contradictorios en el momento de emplear la estrategia de enseñanza por medio de las NTIC, pues en el momento 3 el IHOs empeoró y solo una pequeña porción de los escolares manifestó cambios.

En una revisión elaborada por García et al., se encontró que "los programas efectivos de fomento a la salud, son aquellos que se dirigen a fines múltiples y que son mantenidos en el tiempo suficiente para alcanzar y mantener los objetivos" [18]. Aunque la estrategia empleada con las NTIC constituye una técnica, los resultados obtenidos podrían explicarse por su duración y por la novedad que representa para la educación en salud, lo que implicaría hacer un cambio del paradigma 
de la enseñanza tradicional, en la que el agente de salud es el centro del proceso, a la enseñanza-aprendizaje, en la que cada individuo participa activamente en la construcción de su conocimiento y a través de la experiencia.

Jardines, en su estudio del 2007, plantea que la medicina preventiva y la promoción de la salud serán las que predominen sobre la curativa y las NTIC con respecto a la atención médica tradicional; esto ocasionaría un aumento en el impacto de estrategias como la utilizada en este estudio. Este autor dice también que acceder a este tipo de información forma parte de lo que es la equidad en salud [19].

A pesar de que las NTIC han sido utilizadas en la enseñanza de la salud a nivel universitario, incluyendo el área de la salud, con el tiempo han ingresado a todos los ámbitos del acontecer social y profesional [20]. Se podría suponer que la cultura de la informática tendrá gran predominio en la formación de los futuros adolescentes $[21,22]$, dado que los jóvenes perciben que las tecnologías son necesarias y útiles para la vida, sienten que no se pueden quedar fuera y por esto buscan distintas formas de aprenderlas.

En el contexto actual de la cultura juvenil, las tecnologías pasan a ser un relevante medio de comunicación que se debe aprender [23]. Entre los pocos estudios afines a la educación para la salud bucal y el uso de las NTIC, Franco et al., mediante el diseño y la implementación de una unidad didáctica para concientizar a los estudiantes de secundaria sobre la importancia de la salud y de la higiene bucodental, encontraron que la mayoría de los alumnos son capaces, con las ayudas pertinentes, de valorar adecuadamente la fiabilidad de las fuentes de internet, a la vez que toman conciencia de que el consumo de drogas es perjudicial para su salud [24].

\section{Conclusiones}

La presente investigación es un primer estudio que evalúa los MET y las NTIC en odontología, en el que se usaron índices internacionalmente reconocidos; por lo mismo, es punto de partida para otras intervenciones de este tipo en las que se debe incrementar su duración.

Los resultados obtenidos no se pueden inferir de la población general, dado que los sujetos de estudio fueron seleccionados por conveniencia. Por lo tanto, no se puede establecer causalidad y se deben considerar posibles factores de confusión.
En cuanto a las nuevas alternativas de investigación, se propone indagar sobre el uso de las NTIC empleadas en la educación para la salud, y a partir de los resultados, proponer estrategias que fomenten la apropiación del conocimiento para mejorar la situación de salud de las poblaciones y generar impacto en la calidad de la educación.

Así mismo se propende a motivar a las personas para que desarrollen la capacidad de aprender a aprender y para que sean ellas quienes asuman sus propias responsabilidades, a fin de que sean protagonistas en procesos de educación para la salud a partir de la identificación de sus necesidades. De igual modo, se propende a evaluar las competencias, las habilidades, los conocimientos y las prácticas de los agentes en salud, con el fin de proponer, según los resultados, la implementación de capacitación para los formadores.

Se concluye que sigue existiendo la necesidad de implementar y fortalecer programas educativos para la salud, dirigidos a la población escolarizada de básica primaria y secundaria, con intervenciones que tengan durabilidad y continuidad. Esto con el propósito de mejorar los índices de salud bucal, en particular, y así conseguir un incremento en la calidad de vida, lo cual incluye a las poblaciones vulnerables y especiales, como los niños con discapacidad física y/o cognitiva.

A su vez, también hay la necesidad de proponer un estudio en el que se intervenga a los padres y a los agentes educadores, para que sean ellos los principales promotores del autocuidado y la vigilancia de los escolares en cuanto a la educación para la salud, en especial la salud bucal.

\section{Referencias}

[1] Organización Mundial de la Salud, Ministerio de Salud y Bienestar Social de Canadá, Asociación Canadiense de Salud Pública. Carta de Ottawa para la Promoción de la Salud. Ottawa: OMs; 1986.

[2] Medina-Solís CE, Segovia-Villanueva A, Estrella-Rodríguez R, Maupomé G, Ávila-Burgos L, Pérez-Núñez $\mathrm{R}$. Asociación del nivel socioeconómico con la higiene bucal en preescolares bajo el programa de odontología preventiva del imss en Campeche. Gac Med Mex. 2006;142(5):363-8.

[3] Green JC, Vermillion JR. The Simplified Oral Hygiene Index. J Am Dent Assoc. 1964;68:7-13.

[4] World Medical Association General Assembly. World Medical Association Declaration of Helsinki: Ethical 
Principles for Medical Research Involving Human Subjects. J Int Bioethique. 2004;15(1):124-9.

[5] WHo Expert Committee on Comprehensive School Health Education and Promotion. Promoting Health through Schools [internet]. 1997 [citado 2012 mar 3]. Disponible en: http://whqlibdoc.who.int/trs/WHO TRS 870.pdf

[6] Vigotsky L. Pensamiento y lenguaje: teoría del desarrollo cultural de las funciones psíquicas. Buenos Aires: Fausto; 2012.

[7] Marqués F, Sáez S, Guayta R. Métodos y medios en promoción y educación para la salud. Barcelona: UOC; 2004.

[8] Kwan SY, Petersen PE, Pine CM, Borutta A. Health-Promoting Schools: An Opportunity for Oral Health Promotion. Bull World Health Organ. 2005;83(9):677-85.

[9] Martínez-Herrera E, Agudelo-Suárez AA, GonzálezAriza S. El mercadeo social: una disciplina que fortalece el fomento de la salud bucal en Colombia. Rev CES Odont. 2009;22(2):77-84.

[10] Colgate Palmolive, Centro de Información del Cuidado Bucal y Dental. Guía de cepillado [internet]. s. f. [citado 2013 ago 29]. Disponible en: http://www.colgate.com. uy/app/CP/UY/OC/Information/Interactive-Guides/ Guide-to-Brushing.cvsp

[11] Fundación Pfizer. City Salud. Juego interactivo educación para la salud [internet]. s. f. [citado 2013 ago 29]. Disponible en: www.citysalud.es

[12] Colombia, Ministerio de Salud y la Protección Social. Resolución 8430 de 1993, por la cual se establecen las normas científicas, técnicas y administrativas para la investigación en salud. Bogotá: El Ministerio; 1993.

[13] Reyes W, Grau J, Prendes M de la C. ¿Cómo hacer más efectiva la educación en salud en la atención primaria? Rev Cubana Med Gen Integr. 1999;15(2):176-83.

[14] Muñoz F, López-Acuña D, Halverson P, Guerra C, Hanna W, Larrieu M, et al. Las funciones esenciales de la salud pública: un tema emergente en las reformas del sector de la salud. Rev Panam Salud Pública. 2000;8(1):126-34.

[15] Dávila M, Mujica M. Aplicación de un programa educativo a los escolares sobre enfermedades de la cavidad bucal y medidas preventivas. Acta Odontol Venez. 2008;46(3):1-7.

[16] Torres G, López O, Sardiñas M, Machado R, Pérez L. Intervención educativa para elevar los conocimientos y modificar hábitos sobre salud bucal en niños con retraso mental leve. Universidad Médica de Ciego de Ávila. Mediciego. 2013;19(Supl. 1):1-9.

[17] Freitas O, Zanchett S, Berndt R, Marques M. Motivação no controle do biofilme dental e o aprem relação à saúde bucal em escolares. Biol Saúde Ponta Grossa. 2012;18(2):115-20.

[18] García J, Owen E, Alarcón L. Aplicación del modelo Precede-Proceed para el diseño de un programa de educación en salud. Rev Psicol Salud. 2005;15(1):135-51.

[19] Jardines M. Acceso a la información y equidad en salud. Rev. Cubana Salud Pública. 2007;33(3):1-8.

[20] Moya F. Importancia de las Tic en la enseñanza de salud. Barranquilla: Coruniamericana; 2012.

[21] Servin R, Traverso Y, Solís A, Gómez R. Los adolescentes y la cultura de la informática: un fenómeno creciente. Rev Fac Med Unne. 2013;33(2):36-40.

[22] Observatorio para la Sociedad de la Información en Latinoamérica y el Caribe (Osilac) Cepal/ICA. El estado de las estadísticas sobre Sociedad de la Información en los Institutos Nacionales de Estadística de América Latina y el Caribe. Santiago de Chile: Cepal/ICA; 2004.

[23] Peña S, Borrero I, Marchant A, González P, Novoa G. Percepciones de jóvenes acerca del uso de las tecnologías de información en el ámbito escolar. Última Década. 2006;14(24):37-60.

[24] Franco A, España E, Blanco A, Raón J. Uso de Internet para analizar las relaciones entre drogas y salud bucodental. Una experiencia en $4^{\circ}$ de Educación Secundaria Obligatoria. Málaga: Universidad de Málaga; s. f. 